\title{
Video Game and Fuzzy Logic to Improve Amblyopia and Convergence Insufficiency
}

\author{
Shabnam Sadeghi Esfahlani, Karim Sadeghi Esfahlani, Hassan Shirvani \\ School of Engineering and Built Environment \\ Email: shabnam.sadeghi-esfahlani@anglia.ac.uk \\ esfahlani.k@gmail.com \\ hassan.shirvani@anglia.ac.uk
}

\begin{abstract}
Intuitive learning of visual tasks appears to be an assuring exercise for Amblyopia and Convergence Insufficiency (CI) improvement. Amblyopia is a developmental dysfunction of vision identified by poor monocular visual acuity whereas CI is a common binocular/two-eyed vision disorder in which the eyes do not operate near efficiently. This study proposed the development of virtual reality (VR) video game platform, guided through Fuzzy Logic, targeting casualties with amblyopia and CI condition. The game enables precise control over stimulus parameters, and trains contrast sensitivity with the benefits of motivation and reward that maintain practice over long periods. The dichoptic visual training facilitated through the VR headset and game engine with eye tracking and stimulus data collection capability. This non-invasive eye-training exercise program aims to train the patients to overcome the common conditions such as lazy eye and exophoria at near. Our vision lab made of $2 \mathrm{D}$ and $3 \mathrm{D}$ game environments facilitated exercises, which are recommended by opticians ${ }^{1}$, through three stimulating video game scenarios. The preliminary results of this study have shown that this program has the potential to be adopted for vision therapy. As such in the future study, a randomized clinical trial with participants 9 to 18 years of age will randomly be assigned to receive 12 weeks of vision lab versus home-based pencil push-ups and vision patching exercise for statistical analysis and validation.

Index Terms-Virtual Reality (VR), Amblyopia, Vision Therapy, Convergence Insufficiency
\end{abstract}

\section{INTRODUCTION}

Convergence insufficiency (CI) and amblyopia are vision development condition associated with eye muscle coordination problem and lazy eye, respectively [1]. CI is usually equated with a variety of symptoms including eyestrain, blurred vision, diplopia (double vision), difficulty concentrating, movement of print, and loss of comprehension after short periods of reading or performing close activities due to do not have normal, stable convergence of the two eyes. [2]. Unilateral amblyopia is a loss in visual function in one eye in comparison to the other that force the visual system to prefer one eye over another known as strabismus (cross-eyed) [3]. Strabismus could cause the eyes cross-in (esotropia) or turn-out (exotropia) [4]. Recent studies surveying the ophthalmic community suggest that home-based therapy using pencil push-ups is the most commonly prescribed treatment for young patients with symptomatic CI [5]. The study by [6] implies that two hours of daily patching produces

\footnotetext{
${ }^{1}$ https://nei.nih.gov/
}

an improvement in visual acuity in treating amblyopia in children that is of similar magnitude to the improvement produced by six hours of daily patching. The commonly prescribed treatments are passive treatment with base-in prism reading glasses and active treatments such as home-based therapy using pencil push-ups, office-based vision therapy, and orthoptics [7], [8]. Our VR vision study was structured upon the determinants, and concepts discussed in optometric and ophthalmological textbooks and recommend by vision therapists [9], [10]. The important terms used in vision therapy are; visual acuity (VA), contrast sensitivity (CS), visual plasticity (VP) and perceptual learning (PL).

$\mathrm{VA}$ is the most common clinical measurement of visual function and is considered as the standard measure of visual functions. VA measures the ability to identify black symbols on a white background at a standardized distance as the size of the symbols varies. It could be used to describe several quite different visual tasks; I) Detection acuity: smallest spot or thinnest line that can be seen against a brighter or darker background. II) Localization acuity: smallest visible position shift of a dot or line compared to another dot or line. III) Resolution acuity: the smallest gap between spots or lines that can be seen (a set of parallel lines is called Grating Resolution) and IV) Letter or optotype acuity: smallest letter or another object that can be correctly identified (appendix). $\mathrm{CS}$ is the ability to discriminate between shades of gray, change the effective contrast of the target against the background [11]. CS measurements yield information about an individual's ability to see low-contrast targets over an extended range of target size (and orientation). A CS assessment procedure consists of presenting the player with a sine-wave grating target of a given spatial frequency. The contrast of the target grating is then varied while the observer's contrast detection threshold is determined. Typically, contrast thresholds of this sort are collected using vertically oriented sine-wave gratings varying in spatial frequency from 0.5 (very wide) to 32 (very narrow) cycles per degree of visual angle.

$\mathrm{CSF}$ is the contrast sensitivity function (CSF) describes the combined response of the classical receptive fields of simple cells that have been selectively tuned for location, orientation, and spatial frequency and constitute the fundamental units of analysis [11]. 
VP is the ability of the visual system to change its responses to adapt to changes in the visual input. Evidence for plasticity in the adult visual system has been reported in human studies that have demonstrated that training in specific visual tasks leads to improvement in performance or sensitivity [12].

PL is an experience-dependent enhancement of our ability to make sense of what we see/hear. PL has a significant influence on our understanding of the development and plasticity of the visual system. These changes are permanent or semi-permanent, as distinct from shorter-term mechanisms like sensory adaptation or habituation. Moreover, these changes are not merely incidental but somewhat adaptive and therefore confer benefits, like improved sensitivity to weak or ambiguous stimuli. Improvement after perceptual learning was demonstrated using a variety of visual tasks showing that the adult visual system can change according to behavioral demands [13]. We developed 2D- and 3D-game scenarios (Vision Lab) with the intention to train eyes vision through Convergence, Amblyopia and Dynamic Aim/Peripheral Targeting video game environments. It comprises of exercises integrating both eyes, each viewing an independent stimulus (dichoptic viewing), with the aim of balancing the combination of visual input between the eyes. The main menu is displayed in Fig. 1 (a). Games can be adjusted based on the requirements through its various parameters, including the number of attempts, the difficulty of levels, the number of virtual objects, training the left or right eye, and embedding dichoptic training setting within the objectives and tasks of the game to engage and train the non-dominant eye. The calibration starts with the FOVE's eye and head calibration as the player facing the computers' screen. At the Standard anatomical position, the orientation and position of head and eyes considered to be $(0,0,0)$ where it is in the range of $[-1,1]$. The head position and orientation relative to $\mathrm{x}, \mathrm{y}$ and $\mathrm{z}$-axis identified with $G_{H P(x, y, z)}$, and $G_{H O(x, y, z)}$, respectively. The eyes position calibration start at the centre of the screen $(0,0,0)$ and then the right eye and the left eye are identified via $G_{R E(x, y, z)}$, and $G_{L E(x, y, z)}$, respectively. Game playing duration was denoted as; $G_{P d}$ and game task completion was $G_{T c}$. Amblyopia is a 3D game comprised of a set of marbles (red and black, etc.), which must be classified into two separate bowls based on their colors. The players interact with the virtual marbles using the mouse and keyboard. The dominant eye sees all marbles in black, and the non-dominant eye sees half marbles red half black displayed in Fig. 2 (a,b). The intention is to force the non-dominant eye to be engaged. The screenshot of the game is illustrated in Fig. 1 (b). Some variables in the Amblyopia game could be adjusted before starting the game such as; I) number of marbles $\left(A_{N m}\right)$, II) its colour variety $\left(A_{C m}\right)$ III) environment with distractions/mixed clusters $\left(A_{E d}\right)$, and IV) varying target contrast (to measure basic visual sensitivity) $\left(A_{T c}\right)$.

Convergence is a $2 \mathrm{D}$ video game, that is made up of two matrices/boxed containing randomly generated letters as displayed in Fig. 1 (c). The letters generated in one matrix is identical to the other matrix expect one letter which will be identified through crossing eyes. Through crossed eyes, letters of two matrices overlap (matrices converge). The different letter that is positioned in the same row and column in each matrix stands out. The standout letter must be selected by moving the cursor (using keyboard's arrow keys) and pressing the space bar button to register it and gain scores. The input parameters which could be assigned for the Convergence game are; I) dimension of the letters $\left(C_{D l}\right)$, II) the number of rows and columns of the matrices $\left(C_{N r}\right)$ and $\left(C_{N c}\right)$, III) varying target resolution/contrast $\left(C_{T c}\right)$, and IV) sharpness of the letters $\left(C_{S l}\right)$.

Dynamic Aim 2D-game was made of a rotating disk with randomly generated dots around its center which specified with the letter A known as the target. The player looks/stares at the target (letter A) while clicking on spots using the mouse. The player also must do it as quick as possible to pick as many dots as they could in the specified time duration. The screenshot of the game is shown in Fig. 1 (d). The Dynamic Aim game's input parameters are I) number of dots $\left(D_{N d}\right)$, II $)$ its size $\left(D_{S d}\right)$, III) dimension of the target $\left(D_{D t}\right)$, IV) speed of rotation $\left.\left(D_{S r}\right), \mathrm{V}\right)$ various colours or contrast sensitivity $\left(D_{T c}\right)$ shown in Fig. $2(\mathrm{c}, \mathrm{d})$, and VI) sensitivity of the clicking position $\left(D_{S p}\right)$.

\section{Design And Methodology}

\section{A. FOVE (VR headset)}

FOVE (head mounted display) with the position tracking camera was used which recognizes the LED lights positioned under its cover with Inertial Measurement Units (IMU), Infrared (IR-based) system and the eye-tracking sensor. The headset has (90॰-100॰) field of view, which provides a better game view experience. The sampling rate of the head position tracking is $100 \mathrm{~Hz}$. The display frame rate is $70 \mathrm{~Hz}$, and the eye tracking sensor has $140-180 \mathrm{~Hz}$ sampling rate (120fps). We positioned the position-tracking camera within 3 meters from the player while wearing the headset and interacting with the games. Adaptation of VR headset in the Vision Lab enabled us to collect important data which are necessary for an eye training and improvement system. Our study suggests that it could provide in-home training environment while overcoming the constraints of the traditional in-office training.

\section{B. Fuzzy Logic (FL)}

Fuzzy Logic incorporates human's logic and their reasoning strategy [14], [15]. In the FL, there is a gradual transition from one value to another rather than abrupt change (granulation). There are overlapping fuzzy interval changes, which mimic how human perceive scientific reasoning. FL was incorporated in Vision Lab games to provide a framework for the description of imprecise dependencies and commands through the use of fuzzy if/then or or/and rules. Linguistic variable and values, membership functions, and the universe of discourse are important concepts in the fuzzy set [16]. The fuzzy set is a collection of real numbers having partial membership in 

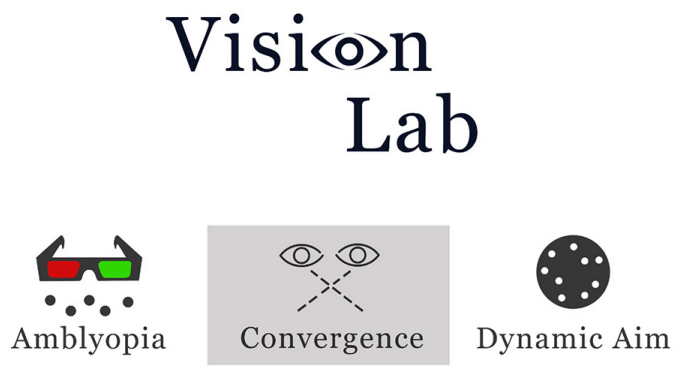

(a)

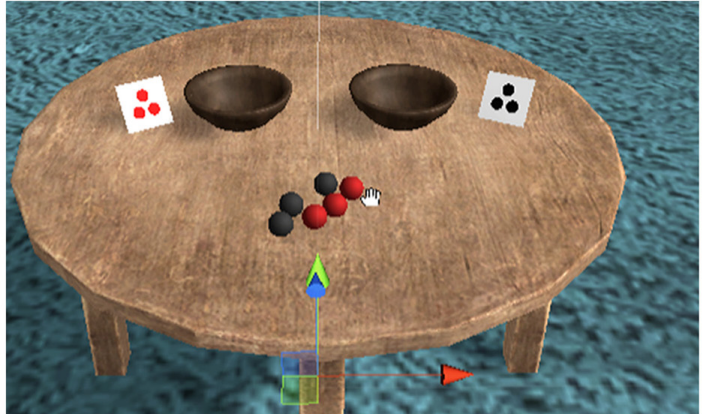

(b)

00:05

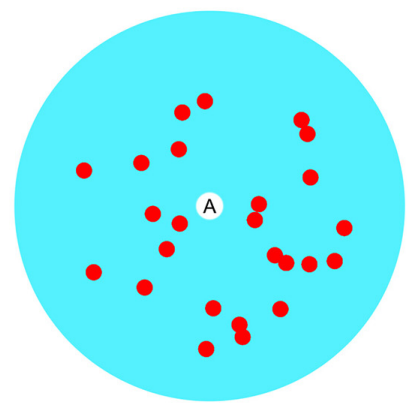

score: 0

(c)

(d)

Fig. 1. Vision Labs three-game scenarios, (a) Main menu, (b) Amblyopia game, (c) Convergence game, (d) Dynamic Aim game.

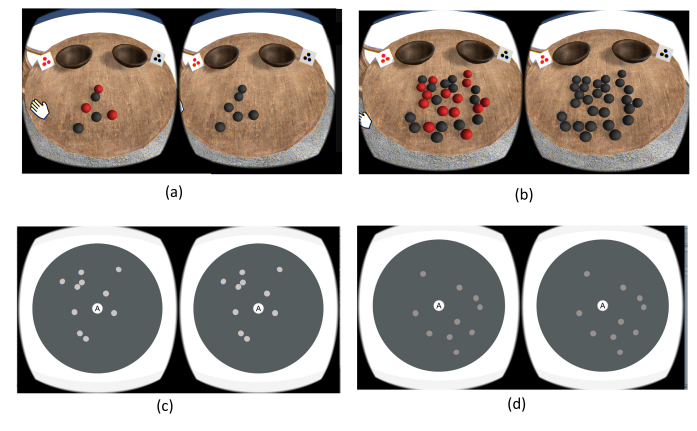

Fig. 2. (a, b) Amblyopia game with the left and right display of FOVE, (c, d) Convergence game with two different shades of gray (CS).

the set. A membership value of 1 specifies the total number of members in the set, absolute exclusion from the game presented with the member value of 0 and partial membership in the set is determined by a membership value between 0 and 1. A membership function (MF) is a curve that defines how each point in the input space is mapped to a membership value in $[0,1]$. The membership function tries to find the standard range for each input and the MF specifies a fuzzy set $A$ as illustrated in Eqn. 1, [17], [18];

$$
\mu: X \rightarrow[0,1] \text { or } A: X \rightarrow[0,1] .
$$

A fuzzy set $A$ in $X$ is directly specified by the function $\left(x, \mu_{A}(x)\right)$, mathematically as follows;

$$
A=\left\{\left(x, \mu_{A}(x)\right) \mid x \in X\right\}
$$

The collection of numbers on which a variable is defined are the universe of discourse for that variable. A variable within a universe of discourse $X$ has a real number $x$ in that $(x \in X)$. A membership function $\mu_{A}(x)$ associated with $A$ is a function that maps $X$ into $[0,1]$ and gives the grade of membership of $X$ as $A$. The MF in the Vision Lab fuzzy logic system was triangular in the range of $[0,1]$ [19]. Eqn defines the triangular membership function. 3 where $\alpha, \beta$, and $\gamma$ are the parameters. The fuzzy logic operates on the input $x$ to produce the crisp output of $\mu$.

$$
\mu_{A}(x: \alpha, \beta, \gamma)=\left\{\begin{array}{cc}
0 & x \leqslant \alpha \\
\frac{x-\alpha}{\beta-\alpha} & \alpha<x \leqslant \beta \\
\frac{\gamma-x}{\gamma-\beta} & \beta \leqslant x<\gamma \\
0 & x \geqslant \gamma
\end{array}\right.
$$

Each triangular function is composed by a set of if - then rules as specified in Eqn. 4.

$$
\begin{cases}\text { If }((x \leqslant \alpha) \wedge(x \geqslant \gamma)) \Rightarrow & f(x)=0 \\ \text { If }((x>\alpha) \wedge(x \leqslant \beta)) \Rightarrow & f(x)=\frac{x-\alpha}{\alpha-\beta} \\ \text { If }((x \geqslant \beta) \wedge(x<\gamma)) \Rightarrow & f(x)=\frac{\gamma-x}{\gamma-\beta}\end{cases}
$$




\section{Vision Lab Game's Mechanism}

The intelligent fuzzy logic inference in the Vision Lab facilitated an exercise which is appropriate to players ability based on their performance and is accompanied by a motivational reward. The normality factor of the training was adopted on input parameters with quantified value that describes how well a player performed in a particular task. Input quantities are standardised and transferred for fuzzification through fuzzy sets. They are sets of predefined rules, applied to fuzzy sets through the fuzzy inference that moves them through defuzzification and denormalization step to generate the crisp output $\mu$. Below is the inputs and output variables integrated in the game through fuzzy logics:

1) Input Variables: The input variables that are used depend on the subjective assessment, game type and the severity of the constraints set in the games. The input variables could be activated or deactivated upon request by the clinician or the player before attempting the game depending on the required constraints and complexity. Three subsets are assigned to the input variables including; Very Good (VG), Good(G), Bad (B).

1) $A_{H P(x, y, z)}, A_{H O(x, y, z)}, A_{R E(x, y, z)}, A_{L E(x, y, z)}, A_{P d}$ and $A_{T c}$.

2) $C_{H P(x, y, z)}, C_{H O(x, y, z)}, C_{R E(x, y, z)}, C_{L E(x, y, z)}, C_{P d}$ and $C_{T c}$.

3) $D_{H P(x, y, z)}, D_{H O(x, y, z)}, D_{R E(x, y, z)}, D_{L E(x, y, z)}, D_{P d}$ and $D_{T c}$.

2) Output: Output is the $G_{P}$ with the subsets of "Progression", "Repetition", and "Simplification". Inputs potentially generate an output which directs the player towards "Progression", (70 - 100)\% "Repetition", $(30-70) \%$ or "Simplification" $(0-30) \%$ calculated based on the average bias against the pre-set constrains. The triangular fuzzy set diagrams are consistent within the constraints of the conditions with small arbitrary error overlaps. It is based on virtual object's position $V o_{P}$ on the screen (target), cursor/mouse positioning $C_{P}$, number of virtual objects $N_{V o}$, and preset game duration $G_{D}$. Linguistic Rules and Constrains: The control rules are implemented using fuzzy conditional statements (if/then, or/and, and not) and the relations between subsets. $A \times B$ (maximum/or), $A+B$ (minimum/and) and $\Gamma A$ (negation/not), Eqn. 5.

$$
\begin{array}{r}
A \times B=\sum_{i} \sum_{j} \min \left\{\mu_{A}\left(u_{i}\right), \mu_{B}\left(\nu_{j}\right)\right\} \\
A+B=\sum_{i} \sum_{j} \max \left\{\mu_{A}\left(u_{i}\right), \mu_{B}\left(\nu_{j}\right)\right\} \\
\Gamma A=1-\sum_{i} \mu_{A}\left(u_{i}\right) \\
\Gamma B=1-\sum_{j} \mu_{B}\left(\nu_{j}\right) \\
i=1,2,3, \ldots, m \\
j=1,2,3, \ldots, n
\end{array}
$$

Where $m$ and $n$ are the numbers of elements in the universes of $A$ and $B$, respectively.

The rules are derived from the Optometry and Visual Science

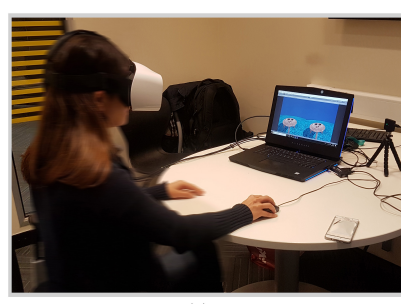

(a)

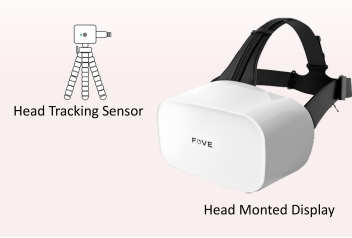

(b)
Fig. 3. (a) The subject playing the Amblyopia game. (b) FOVE head mounted display head tracking sensor.

[20] and opticians consultancy and collaboration. Considering the pupillary distance (PD) which is the distance measured in millimeters between the centers of the pupils of the eyes [21], and monocular PD which refers to the distance between each eye and the bridge of the nose [20]. It was expected that the $G_{L E(x)}$ and $=G_{R E(x)}$ position on the screen have an average distance equal to PD in ( $\mathrm{mm}$ ). The average adults PD is between 54-74 mm and kids' are between 43-58 $\mathrm{mm}$. This value is measure through the FOVE's initial calibration and was used to set the constrains in the fuzzy sets. We expect this value do net exceed 74 for adults and 58 for kids which may be slightly different for each eye due to anatomical variations. Any deviation will be measure as error and used for fuzzification. The head's orientation ( $-90 \circ$ to $90 \circ)$ and position is measured relative to the standard anatomical positioning and biases calculated against this standards. The fuzzy constrains are specified an follows output is calculated based on input constrains and the responding results which structured as follows;

$$
\begin{aligned}
& \text { If } \operatorname{Vop}_{P} \text { is...and/or } C_{P} \text { is...then } \\
& G_{R E(x, y, z)} \text { and } G_{L E(x, y, z)} \text { is... } \\
& \text { If } V o_{P} \text { is...and/or } C_{P} \text { is...then } \\
& G_{H O(x, y, z)} \text { and } H P_{L E(x, y, z)} \text { is... } \\
& \text { If } N_{V O} \text { is...and/or } G_{D} \text { is...then ... }
\end{aligned}
$$

\section{RESUlts AND Discussion}

\section{A. Results}

The institution certified ethics approval and consent form were provided. Three participants volunteered to play the game; 2 male and one female with the mean age of twentyone. Two participants with the lazy eye condition and a participant with CI. The study was performed for one hour in each session for eight consecutive weeks. Before starting the data collection, we described the study procedure to the participants and asked them to read and sign the informed consent form. The participants were provided with the openended questionnaire at the end of each session.

The fuzzy inference was coded in Unity3D through a fuzzyplugin. The fuzzy inference process was applied at the level of individual rules. The contribution of each fuzzy set inferred was considered individually, and the final crisp control action is obtained by taking a calculus (an average) over a concrete 


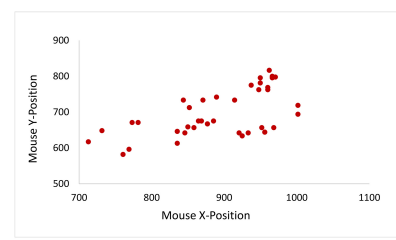

(a)

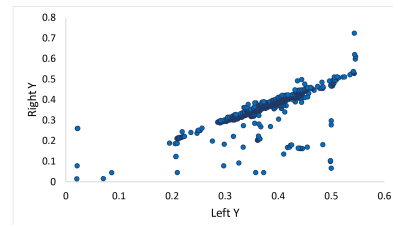

(c)

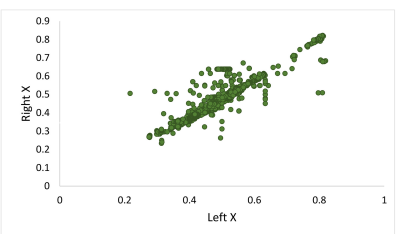

(b)

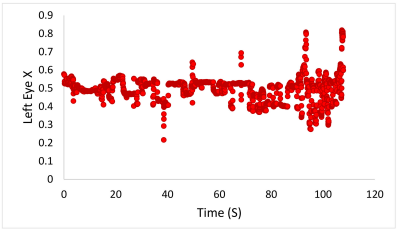

(d)
Fig. 4. (a) The mouse click positions on the screen. (b, c) Left and right eye positions on the screen relative to each other. (d) Left eye $\mathrm{x}$ position relative to time.

fresh characteristic value from inputs.

The results and comments collected from the participants showed that the Vision Lab is capable of providing a customized, engaging and computer supervised platform for eye improvement condition which could help with visual plasticity. Simple reaction time was measured as target detection (detection of targets at various color contrasts/locations) in a criterion where the timing, location, and shades of the visual target vary randomly where detection rates and latency were quantified. From the results, we determined that the subject's reaction time improved linearly with increased contrast of the targets. Participants with a better vision could detect the target and react quicker to the stimuli compared to other participants (with lower vision). Fig. 4 displays the data collected from the headset while players were interacting with the Vision Lab. Fig. 4 (a) plots the mouse click positions on the screen. Fig. 4 (b, c) displays left, and right eye positions on the screen relative to each other and (d) shows left eye's $x$ position relative to time. However, our study has some limitations due to the limited number of participants and lacks validation which will be addressed in our future works.

\section{B. conclusion}

Amblyopia infirmity (lazy eye) could result from any condition that prevents the eye from focusing. It could result in misalignment of the two eyes known as strabismus where the eyes cross in (esotropia) or turn out (exotropia) ${ }^{2}$. The earlier the patient receives therapy the better the outcome would be. Unilateral amblyopia is a loss in visual function in one eye in comparison to the other and is often caused by other associated factors that force the visual system to prefer one eye over another. The most common of these factors is a difference in refractive error between the two eyes, usually in spherical correction (anisometropic amblyopia) and strabismus [1], [4]. Many other forms of unilateral amblyopia occur as a result of pathological changes in the structure in or

\footnotetext{
${ }^{2}$ https://nei.nih.gov/
}

around the eye such as unilateral cataracts or ptosis (stimulus deprivation amblyopia) [22]. Convergence insufficiency (CI) is the inability to maintain fusion on a near target. It is the leading cause of eyestrain, blurred vision, double vision (diplopia), headaches, asthenopia, and difficulty reading or performing tasks at near [23]. Visual acuity, contrast sensitivity, and other visual judgments are reduced in the affected eye, and binocular function is degraded or absent [1], [4]. Home-based pencil push-ups therapy appears to be the most commonly prescribed treatment, but scientific studies have shown that this treatment is ineffective. If the eyes do drift out, the person is likely to have double vision. To prevent double vision, the individual exerts extra effort to make the eyes turn back in (converge). This extra effort can lead to some frustrating symptoms which interfere with the ability to read and work comfortably at near. Many people who would test as having convergence insufficiency [if tested] may not complain of double vision or the other symptoms listed above because the vision in one eye has shut down. In other words, even though both eyes are open and are healthy and capable of sight, the person's brain ignores one eye to avoid double vision. This is a neurologically active process called suppression. Suppression of view in one eye causes loss of binocular (two-eyed) vision and depth perception. Poor binocular vision can harm many areas of life, such as coordination, sports, judgment of distances, eye contact, or motion sickness. The standard treatments for amblyopia are penalizing therapies, such as patching or blurring vision with atropine which forces the use of the amblyopic eye. This characteristic is replaced through the double screen vision of the VR headset, which provides separate stimuli to each eye. In conclusion, we should highlight that the individualized and automatically supervised platform could offer a home-based solution in that the parameters including the size, exposure duration, contrast, and adaptation level, patients progress, etc., was leveraged through the Fuzzy logic. The increasing availability of 'offthe-shelf' gaming peripherals in the consumer market has provided more significant opportunities for research into nongaming or serious gaming applications. The Unity 3D game engine facilitates a fuzzy logic solution incorporated in a virtual environment. The intelligent system can halt game progression and game difficulty until the player achieves a desired outcome. The system offers the possibility to provide a personalized, autonomously-learned rehabilitation program for patients.

\section{REFERENCES}

[1] D. M. Levi and U. Polat, "Neural plasticity in adults with amblyopia," Proceedings of the National Academy of Sciences, vol. 93, no. 13, pp. 6830-6834, 1996.

[2] Á. García-Munoz, S. Carbonell-Bonete, and P. Cacho-Martínez, "Symptomatology associated with accommodative and binocular vision anomalies," Journal of Optometry, vol. 7, no. 4, pp. 178-192, 2014.

[3] G. Campana and M. Maniglia, Learning to see (better): Improving visual deficits with perceptual learning. Frontiers Media SA, 2015.

[4] L. Kiorpes, S. P. McKee, et al., "Neural mechanisms underlying amblyopia," Current opinion in neurobiology, vol. 9, no. 4, pp. 480-486, 1999. 
[5] M. Scheiman, J. Cooper, G. L. Mitchell, P. De Land, S. Cotter, E. Borsting, R. London, and M. Rouse, "A survey of treatment modalities for convergence insufficiency," Optometry and vision science, vol. 79, no. 3, pp. 151-157, 2002.

[6] M. X. Repka, R. W. Beck, J. M. Holmes, E. E. Birch, D. L. Chandler, S. A. Cotter, R. W. Hertle, R. T. Kraker, P. S. Moke, G. E. Quinn, et al., "A randomized trial of patching regimens for treatment of moderate amblyopia in children.," Archives of ophthalmology (Chicago, Ill.: 1960), vol. 121, no. 5, pp. 603-611, 2003.

[7] C. INSUFFI, "Randomized clinical trial of treatments for symptomatic convergence insufficiency in children," Arch Ophthalmol, vol. 126, no. 10, pp. 1336-1349, 2008.

[8] K. J. Ciuffreda, "The scientific basis for and efficacy of optometric vision therapy in nonstrabismic accommodative and vergence disorders," OPTOMETRY-ST LOUIS-, vol. 73, no. 12, pp. 735-762, 2002.

[9] N. R. Council et al., Emergent techniques for assessment of visual performance. National Academies, 1985.

[10] T. Grosvenor and T. P. Grosvenor, Primary care optometry. Elsevier Health Sciences, 2007.

[11] U. Polat, "Making perceptual learning practical to improve visual functions," Vision research, vol. 49, no. 21, pp. 2566-2573, 2009.

[12] M. Fahle, T. Poggio, T. A. Poggio, et al., Perceptual learning. MIT Press, 2002

[13] Y. Sasaki, J. E. Nanez, and T. Watanabe, "Advances in visual perceptual learning and plasticity," Nature Reviews Neuroscience, vol. 11, no. 1, p. 53, 2010 .

[14] J. H. Lilly, Fuzzy control and identification. John Wiley \& Sons, 2011.

[15] L.-X. Wang, "A course in fuzzy systems and control prentice hall," Facsimile edition, 1997.

[16] L. A. Zadeh, "The role of fuzzy logic in modeling, identification and control," 1994.

[17] C. MacDonald, Z. Moussavi, and T. Sarkodie-Gyan, "Formation of an internal model of environment dynamics during upper limb reaching movements: A fuzzy approach," in 2007 29th Annual International Conference of the IEEE Engineering in Medicine and Biology Society, pp. 4862-4865, IEEE, 2007.

[18] E. Czogala and J. Leski, Fuzzy and neuro-fuzzy intelligent systems, vol. 47. Physica, 2012.

[19] S. S. Esfahlani, S. Cirstea, A. Sanaei, and G. Wilson, "An adaptive self-organizing fuzzy logic controller in a serious game for motor impairment rehabilitation," in Industrial Electronics (ISIE), 2017 IEEE 26th International Symposium on, pp. 1311-1318, IEEE, 2017.

[20] D. S. McCleary, The Optician Training Manual 2nd edition: Simple Steps to Becoming a Great Optician. Santa Rosa Publishing, 2018.

[21] M. Millodot, Dictionary of Optometry and Visual Science E-Book. Elsevier Health Sciences, 2014.

[22] J. M. Holmes, R. W. Beck, M. X. Repka, D. A. Leske, R. T. Kraker, R. C. Blair, P. S. Moke, E. E. Birch, R. A. Saunders, R. W. Hertle, et al., "The amblyopia treatment study visual acuity testing protocol," Archives of ophthalmology, vol. 119, no. 9, pp. 1345-1353, 2001.

[23] J. B. Lavrich, N. J. Warner, A. J. Hauschild, A. Thau, B. N. Wasserman, C. DeBenedictis, B. Schnall, D. Manley, K. Gunton, S. Padidam, et al., "Inconsistent diagnostic criteria for convergence insufficiency," Journal of American Association for Pediatric Ophthalmology and Strabismus, 2019.

[24] H. Weinberger, "Conjecture on the visual estimation of relative radial motion," Nature, vol. 229, no. 5286, p. 562, 1971

[25] M. E. Maddox and A. Kiefer, "Looming threshold limits and their use in forensic practice," in Proceedings of the Human Factors and Ergonomics Society Annual Meeting, vol. 56, pp. 700-704, Sage Publications Sage CA: Los Angeles, CA, 2012

\section{APPENDIX}

\section{A. Spatial Resolution}

Visual acuity is a measure of the spatial resolution of the visual processing system. VA, is tested by requiring the person whose vision is being tested to identify so-called optotypes stylized letters, Landolt rings, pediatric symbols, symbols for the illiterate, standardized Cyrillic letters in the GolovinSivtsev table, or other patterns on a printed chart (or some other means) from a set viewing distance. Optotypes are represented as black symbols against a white background (i.e., at maximum contrast). The distance between the person's eyes and the testing chart is set to approximate optical infinity in the way the lens attempts to focus (far acuity) or at a defined reading distance (near acuity) ${ }^{3}$. Visual acuity is considered normal at $6 / 6$ vision, equivalent to $20 / 20$ vision (6 meters/20 feet), a human eye with that performance can separate contours that are approximately $1.75 \mathrm{~mm}$ apart. Healthy individuals have an acuity of $6 / 4$ or better (depending on age and other factors). In the expression, $6 / x$ vision, the numerator 6 is the distance in meters between the subject and the chart and the denominator is the distance at which a person with $6 / 6$ acuity would discern the same optotype. Thus, $6 / 12$ means that a person with $6 / 6$ vision would recognize the same optotype from 12 meters away. This is equivalent to saying that with $6 / 12$ vision, the person possesses half the spatial resolution and needs twice the size to discern the optotype. A simple and efficient way to state acuity is by solving the fraction to a decimal number. $6 / 6$ then corresponds to an acuity (or a Visus) of 1.0. The precise distance at which acuity is measured is not important as long as it is sufficiently far away and the size of the optotype on the retina is the same. That size is specified as a visual angle, which is the angle, at the eye, under which the optotype appears. For $6 / 6=1.0$ acuity, the size of a letter on the Snellen chart or Landolt $\mathrm{C}$ chart is a visual angle of 5 arc minutes $(1 \operatorname{arc} \min =1 / 60$ of a degree $)$. By the design of a typical optotype, the critical gap that needs to be resolved is $1 / 5$ this value;

$$
\text { acuity }=\frac{1}{\text { gap size }[\operatorname{arc~} \min ]}
$$

\section{B. Value of Visual Acuity Measurements}

The value of visual acuity measurements is well proven for correcting refractive errors. In the past two decades, a universal language emerged for assessing vision known as the measurement of the contrast sensitivity function which complements visual acuity [9]. The most commonly used definition of contrast is:

$$
\frac{L_{\max }-L_{\min }}{L_{\max }+L_{\min }}
$$

Where $L_{\max }$ is the maximum luminance at the peak and $L_{\min }$ is the minimum luminance at the trough of the sine wave. Contrast may vary from 0.0 (a field of uniform luminance) to 1.0 (where $L_{\min }=0.0$ and $L_{\max }=2.0 * L_{\text {mean }}$ since $\left.L_{\text {mean }}=\frac{\left.L_{\max }+L m i n\right)}{2}\right)$. It is not possible to have a real sinusoidal grating of contrast greater than 1.0 because luminance cannot be less than 0.0. The mean luminance of the sinusoidal gratings has a profound effect on the contrast sensitivity function. At high photopic levels of mean luminance, the normal contrast sensitivity function has a peak sensitivity at about five cpd and a high-frequency cutoff at about $60 \mathrm{cpd}$. As mean luminance is lowered, not only do the frequencies of the peak sensitivity and the high-frequency cutoff become lower, but also the height of the peak is reduced. At mesopic levels of

\footnotetext{
${ }^{3}$ https://en.wikipedia.org/wiki/Visual_acuity
} 
mean luminance, the peak in the contrast sensitivity function has practically disappeared. This peak, which is so prominent at high luminance, is generally believed to reflect the dynamic interaction between excitatory and inhibitory influences in the visual system.

The eye has acuity limits for detecting motion. Forward motion is limited by the subtended angular velocity detection threshold (SAVT), and lateral motion thresholds limit horizontal and vertical motion acuity. The lateral motion limit is generally below the looming motion limit, and for an object of a given size, lateral motion becomes the more insightful of the two, once the observer moves sufficiently far away from the path of travel. Below these thresholds subjective constancy is experienced following the Stevens' power law and WeberFechner law ${ }^{4}$.

\section{Subtended Angular Velocity Detection Threshold (SAVT)}

There is a specific acuity limit in detecting an approaching object's looming motion [24]. This is regarded as the SAVT, limit of visual acuity. It has a practical value of $0.0275 \mathrm{rad} / \mathrm{s}$ [25]. For a person with SAVT $\operatorname{limit}^{5}$ of $\dot{\theta}_{t}$ the looming motion of a directly approaching object of size $S$, moving at velocity $v$, is not delectable until its distance $D$ is;

$$
D \lesssim \sqrt{\frac{S \cdot v}{\dot{\theta}_{t}}-\frac{S^{2}}{4}},
$$

where the $S^{2} / 4$ term is omitted for small objects relative to great distances by small-angle approximation. In order to exceed the SVAT, an object of size $S$ moving as velocity $v$ must be closer than $D$; beyond that distance, subjective constancy is experienced. The SVAT $\dot{\theta}_{t}$ can be measured from the distance at which a looming object is first detected:

$$
\dot{\theta}_{t} \approx \frac{4 S \cdot v}{S^{2}+4 D^{2}}
$$

where the $S^{2}$ term is omitted for small objects relative to great distances by small-angle approximation. The formula is derived from taking the derivative of the visual angle with respect to distance, and then multiplying by velocity to obtain the time rate of visual expansion $(d / d t=d / d x \cdot d x / d t)$.

\section{Lateral motion}

There are acuity limits $\left(\dot{\theta}_{t}\right)$ of horizontal and vertical motion as well ${ }^{6,7}$. They can be measured and defined by the threshold detection of movement of an object traveling at distance $D$ and velocity $v$ orthogonal to the direction of view, from a set-back distance $B$ with the formula

$$
\dot{\theta}_{t} \approx \frac{B \cdot v}{B^{2}+D^{2}}
$$

Because the tangent of the subtended angle is the ratio of the orthogonal distance to the set-back distance, the angular time

\footnotetext{
${ }^{4}$ https://en.wikipedia.org/wiki/Stevens $\backslash \% 27$ s_power_law

${ }^{5}$ https://www.ncbi.nlm.nih.gov/books/NBK219049/

${ }^{6}$ https://jov.arvojournals.org/article.aspx? articleid $=2122525$

${ }^{7}$ https://www.opt.uh.edu/onlinecoursematerials/stevenson5320/L08Acuity.pdf
}

rate $(\mathrm{rad} / \mathrm{s})$ of lateral motion is simply the derivative of the inverse tangent multiplied by the velocity $(d / d t=d / d x \cdot d x / d t)$. In application this means that an orthogonally traveling object will not be discernible as moving until it has reached the distance

$$
D \lesssim \sqrt{\frac{B \cdot v}{\dot{\theta}_{t}}-B^{2}}
$$

where $\dot{\theta}_{t}$ for lateral motion is generally $\geqslant 0.0087 \mathrm{rad} / \mathrm{s}$ with probable dependence on deviation from the fovia and movement orientation, velocity is in terms of the distance units, and zero distance is straight ahead.

\section{E. Radial motion}

The motion acuity limit affects radial motion in accordance to its definition, hence the ratio of the velocity $v$ to the radius $R$ must exceed $\dot{\theta}_{t}$ :

$$
\dot{\theta}_{t} \lesssim \frac{v}{R}
$$

Radial motion is encountered in clinical, research environments, and in virtual-reality headsets. 